\title{
Cardiac biomarkers by point-of-care testing - back to the future?
}

https://doi.org/10.1515/labmed-2019-0180

Received October 23, 2019; accepted January 16, 2020; previously published online February 12, 2020

Abstract: The measurement of the cardiac troponins (cTn), cardiac troponin $\mathrm{T}$ (cTnT) and cardiac troponin I (cTnI) are integral to the management of patients with suspected acute coronary syndromes (ACS). Patients without clear electrocardiographic evidence of myocardial infarction require measurement of $\mathrm{cTnT}$ or cTnI. It therefore follows that a rapid turnaround time (TAT) combined with the immediacy of results return which is achieved by point-of-care testing (POCT) offers a substantial clinical benefit. Rapid results return plus immediate decisionmaking should translate into improved patient flow and improved therapeutic decision-making. The development of high sensitivity troponin assays offer significant clinical advantages. Diagnostic algorithms have been devised utilising very low cut-offs at first presentation and rapid sequential measurements based on admission and $3 \mathrm{~h}$ sampling, most recently with admission and $1 \mathrm{~h}$ sampling. Such troponin algorithms would be even more ideally suited to point-of-care testing as the TAT achieved by the diagnostic laboratory of typically $60 \mathrm{~min}$ corresponds to the sampling interval required by the clinician using the algorithm. However, the limits of detection and analytical imprecision required to utilise these algorithms is not yet met by any easy-to-use POCT systems.

Keywords: analytical performance; cardiac troponin I; cardiac troponin T; myocardial infarction; point-of-care testing.

\section{Introduction}

The role of cardiac biomarker testing using point-ofcare testing (POCT) has to be seen in the context of three

\footnotetext{
*Correspondence: Dr. Paul Collinson, Departments of Clinical Blood Sciences and Cardiology, St George's University Hospitals NHS Foundation Trust and St George's University of London, Cranmer Terrace London SW17 0QT, UK, Phone: 02087255934 Fax: 0208725 5838, E-Mail: paul.collinson@stgeorges.nhs.uk
}

factors. First, the changes that have occurred in the management strategy for patients presenting with suspected ischaemic heart disease. Second, the changing role of cardiac biomarker measurement in the diagnosis and management of patients with suspected acute coronary syndrome (ACS). Finally, the paradigm shift that has occurred following development of assays for the cardiac troponins (cTn) cardiac troponin $\mathrm{T}(\mathrm{cTnT}$ ) and cardiac troponin I (cTnI). Initially the diagnosis of acute myocardial infarction (AMI) was based on clinical assessment and the electrocardiogram (ECG). The role of cardiac biomarkers was recognised in the original World Health Organization (WHO) definition of AMI but only for confirmation of diagnosis when definitive ECG changes were not present [1]. In addition, the diagnostic discriminant used was twice the upper reference limit of the biomarker in question. In statistical terms this meant that the probability of a positive result occurring in a patient without AMI was very low, $0.0044 \%$. Thus, the probability of a false positive diagnosis arising from biochemical testing was very small indeed. This expectation of a very low false positive rate from biochemical testing has had a profound impact when biochemical testing assumed a more central role.

\section{Changing management strategies in patients with acute chest pain}

The diagnosis of AMI in patients with chest pain has undergone a series of step changes. Initially it was considered that coronary artery was universally fatal and it was not until the observation by Herrick in 1912 that myocardial infarction was considered to be survivable. Thereafter however, management was supportive and active intervention was considered but not universally accepted. The demonstration that intracoronary thrombosis was the underlying pathophysiological basis of myocardial infarction led to a reappraisal. Strategies based on opening the coronary artery by thrombolysis and by acute catheterisation were investigated and have become standard of care. Indeed, cardiac catheterisation and stent placement form

Ә Open Access. (C) 2020 Paul Collinson, published by De Gruyter. (cc) BY Public License. 
the basis of current guidelines [2, 3]. The initial strategy of serial measurement of cardiac biomarkers on a daily basis served only to retrospectively confirm diagnosis. Interest developed in more rapid strategies partly to allow earlier identification of patients with AMI and also to identify patients without AMI who could be rapidly and safely discharged from the hospital. This trend was largely driven by the development of chest pain units and recognition that the majority of patients presenting with chest pain did not have AMI. The economics of inappropriate admission of patients with chest pain where the final diagnosis excluded AMI was recognised combined with a desire not to discharge patients inappropriately. This was most well developed in the US [4]. Diagnosis based on rapid serial measurements of myoglobin, creatine kinase (CK) and its MB isoenzyme (creatine kinase myocardial band [CK-MB]) were developed including the concept of delta change. Initial developments in POCT mirrored this with development of technologies, initially single analytes and subsequently panels of cytoplasmic markers. Measurement of cardiac biomarkers shifted from ancillary test to part of the strategy of patient assessment combined with clinical history and the ECG.

\section{Troponin assays and the paradigm shift in the definition of myocardial infarction}

The development of immunoassays for cardiac structural proteins specifically cTnI and cTnT led to a number of studies to evaluate the additional clinical value of $\mathrm{cTn}$ measurement. When compared with diagnosis utilising CK or CK-MB, cTn had two distinct advantages. First, they identified a subset of patients with an apparent diagnosis of unstable angina based on conventional criteria that had an adverse prognosis [5, 6]. Second, they were specific for myocardial injury in the presence of other elevations of CK or CK-MB. The net result was that troponin was viewed as a test specific for AMI and used for dichotomous classification of AMI/No AMI and gained wide acceptance.

There were two significant flaws with this approach. The measurement of cTn was being compared against diagnosis based on WHO criteria. This meant that in patients without definitive ECG changes, the comparative diagnostic gold standard was a CK-MB twice the upper reference limit, with the extremely low false positive rate as discussed above. When this is combined with the complete cardiospecificity of cTn, the radically improved clinical diagnostic sensitivity was unsurprising. The second flaw was the relative insensitivity of the troponin assay in use at that time. These assays were unable to detect troponin in normal individuals and only detected significant elevations largely occurring in patients with AMI.

In addition to the consistent detection of high-risk individuals missed by conventional cardiac enzymes, a series of studies demonstrated that the detection of an elevated troponin in patients considered to have unstable angina predicted the response to low molecular weight heparin [7], glycoprotein IIb/IIIa antagonists [8] and revascularisation procedures [9]. These clear advantages led to the widespread adoption of cTn as the cardiac biomarker of choice. The true paradigm shift came with a series of expert conferences [10] culminating in the redefinition of myocardial infarction using cTnT and cTnI as the gold standard test for the detection of AMI [11, 12].

These changes were reflected in the evolution of POCT with the development of marker panels including cTn. The logic behind this was the concept of a temporal period of "troponin blindness". Hence, cytoplasmic markers, typically myoglobin and CK-MB were combined with cTn [13]. The idea was that the cytoplasmic markers would be released early and provide diagnostic sensitivity whereas the cTn would be detected subsequently and provide specificity of diagnosis. In addition, POCT tests for cTnT [14] and cTnI [15] alone were developed and showed comparable diagnostic sensitivity with central laboratory testing (CLT) but with markedly improved turnaround time (TAT). The crucial component to recognise at this point is that POCT was diagnostically equivalent to CLT. Diagnostic and management pathways could therefore use POCT. The challenge was to demonstrate clinical utility.

\section{POCT for rapid diagnosis in patients with chest pain to improve patient management - what is the evidence base for clinical effectiveness?}

It is an article of faith that rapid provision of test results by POCT will improve patient flow, reduce costs and improve the patient experience. The differential diagnosis of patients presenting with chest pain would seem to be an ideal situation where this could be applied. The management pathways for patients with myocardial infarction are well defined. Patients presenting 
with characteristic changes on the ECG, ST elevation, are characterised as ST elevation myocardial infarction (STEMI) [3]. Management is by immediate revascularisation ideally by coronary angiography and stent placement or if this is not possible by thrombolysis followed by subsequent angiography. Patients without definitive ECG changes require a more sophisticated workup involving biochemical testing. It is here that troponin measurement defines the subsequent management pathways. Patients with an acute troponin elevation are categorised as probable non-ST elevation myocardial infarction (NSTEMI) and are managed according to current treatment guidelines [2]. One important part of this process is that in the majority of patients immediate catheterisation is not required and repeat biochemical testing to confirm the diagnosis can be performed. In patients presenting without definitive ECG changes only a minority will have a final diagnosis of NSTEMI and biochemical testing is the cornerstone of management [16]. It would therefore seem ideal that is performed by POCT.

Studies on POCT in patients presenting with chest pain/AMI have tended to be observational in nature.
The definitive answer to whether or not POCT would be of value would be achieved by a randomised controlled trial. Data from the randomised controlled trials of POCT are summarised in Table 1. Although the results have not always been entirely consistent, the general trend is that POCT results in reduction in length of patient stay and improved throughput. However, it is a common finding in the studies that impact on length of stay is critically dependent on process of care. Indeed this was specifically commented on in two of the studies $[18,20]$. In one comparison the length of stay in the participating six hospitals showed radically different results [25]. The economic consequences of cardiac POCT have been relatively less examined. The Randomised Assessment of Treatment using Panel Assay of Cardiac markers (RATPAC) trial included economic modelling and costing $[21,22]$. They concluded that POCT was not cost-effective largely because the major savings made were in Emergency Department (ED) length of stay (which is low cost) but there was an increase in admissions to the hospital and coronary care unit. In this trial there was statistically significant improvement in accuracy of diagnosis of patients with AMI which accounted for the increased

Table 1: Clinical trials of point-of-care testing.

\begin{tabular}{|c|c|c|c|c|c|c|}
\hline Type & Methodology & Cut off & Location & Outcome & Result & Author \\
\hline Single centre RCT & $\begin{array}{l}\text { Roche CTnT CLT } \\
\text { vs. POCT }\end{array}$ & $0.2 \mu \mathrm{g} / \mathrm{L}$ & $\begin{array}{l}\text { ED referrals } \\
\text { to } C C U\end{array}$ & $\begin{array}{l}\text { Length of stay in } \\
\text { pre-specified rule } \\
\text { out subgroup }\end{array}$ & $\begin{array}{l}\text { Reduction of length of } \\
\text { stay in POCT group }\end{array}$ & Collinson et al. [17] \\
\hline Single centre RCT & $\begin{array}{l}\text { Stratus CS vs. } \\
\text { Dimension RxL }\end{array}$ & $0.1 \mu \mathrm{g} / \mathrm{L}$ & ED & $\begin{array}{l}\text { Time to treatment } \\
\text { Length of stay in } \\
\text { the ED }\end{array}$ & $\begin{array}{l}\text { Reduced time to } \\
\text { treatment } \\
\text { No reduction in ED stay }\end{array}$ & Renaud et al. [18] \\
\hline $\begin{array}{l}3 \text { Centres prospective } \\
\text { observational study }\end{array}$ & $\begin{array}{l}\text { Triage cardiac } \\
\text { panel vs. } \\
\text { Dimension RxL }\end{array}$ & $0.4 \mu \mathrm{g} / \mathrm{L}$ & ED & $\begin{array}{l}\text { Retrospective } \\
\text { review of diagnostic } \\
\text { accuracy } \\
\text { Length of stay }\end{array}$ & $\begin{array}{l}\text { Improved diagnostic } \\
\text { accuracy of multimarker } \\
\text { strategy } \\
\text { Increased number } \\
\text { of discharges }<24 \mathrm{~h} \\
\text { compared with historical } \\
\text { data }\end{array}$ & Straface et al. [19] \\
\hline $\begin{array}{l}2 \text { Centre Cluster } \\
\text { randomised } \\
\text { controlled trial }\end{array}$ & $\begin{array}{l}\text { iStat vs. } \\
\text { Beckman } \\
\text { Coulter Accu I }\end{array}$ & ns & ED & Length of ED stay & Not significant & Loten et al. [20] \\
\hline 6 Centre RCT & $\begin{array}{l}\text { Stratus CS vs. } \\
\text { Central Lab }\end{array}$ & $0.7 \mu \mathrm{g} / \mathrm{L}$ & ED & $\begin{array}{l}\text { Discharges }<4 \mathrm{~h} \\
\text { Length of hospital } \\
\text { stay } \\
\text { MACE }\end{array}$ & $\begin{array}{l}\text { Increased discharge }<4 \mathrm{~h} \\
\text { with less admissions } \\
\text { MACE was equivalent in } \\
\text { POCT and CLT groups }\end{array}$ & $\begin{array}{l}\text { Goodacre et al. } \\
{[21,22]}\end{array}$ \\
\hline Cluster RCT & $\begin{array}{l}\text { Roche cardiac } \\
\text { reader }\end{array}$ & $0.1 \mu \mathrm{g} / \mathrm{L}$ & $\begin{array}{l}68 \text { Rural } \\
\text { primary care } \\
\text { centres }\end{array}$ & Clinical diagnosis & $\begin{array}{l}\text { Improved diagnostic } \\
\text { accuracy }\end{array}$ & $\begin{array}{l}\text { Tomonaga et al. } \\
\text { [23] }\end{array}$ \\
\hline Single centre RCT & $\begin{array}{l}\text { AQT Flex vs. hs } \\
\text { cTnT }\end{array}$ & $14 \mathrm{ng} / \mathrm{L}$ & ED & MACE at 3 months & No significant differences & Asha et al. [24] \\
\hline
\end{tabular}

RCT, randomised controlled trial; cTnT, cardiac troponin T; cTnl, cardiac troponin I; ED, emergency department; CCU, Coronary Care Unit; POCT, point-of-care testing; CLT, central laboratory testing; MACE, major adverse coronary events; ns, not stated. 
admissions. It is arguable that the improved diagnostic accuracy from POCT would offset the apparent minimal change in costs.

\section{Evolution of diagnostic strategies for rapid diagnosis of patients presenting with chest pain unsuspected AMI - high sensitivity cardiac troponin assays}

The recommended diagnostic strategy for patients presenting with chest pain was to measure cardiac troponin on admission and $6 \mathrm{~h}$ later. This strategy reflected the diagnostic performance of cardiac troponin assays whether by POCT or CLT. However, there has been progressive improvement in the analytical performance of cardiac troponin assays culminating in the development of high sensitivity cardiac troponin measurement. This has been driven by two factors. First, all manufacturers work to improve assay performance on a regular basis. The objective is to improve analytical imprecision at the low end of the measuring range. For cTn measurement, an additional incentive has been a consequence of the redefinition of myocardial infarction in terms of troponin. In the first document proposing redefinition of myocardial infarction with cTn as the preferred cardiac biomarker it is specifically stated [11]

"Acceptable imprecision (coefficient of variation) at the $99^{\text {th }}$ percentile for each assay should be defined as $<10 \%$ ".

Since when this was published none of the commercially available cTn assays met these criteria, there was pressure on the manufacturers to improve analytical sensitivity. Initially, assays met the imprecision goal but would only detect cTn in $10-20 \%$ of normal healthy individuals. These have been designated as contemporary sensitive assays. The ultimate goal has been to produce assays that would measure cTn in the majority of normal healthy individuals. A high sensitivity troponin assay has been defined by the International Federation of Clinical Chemists (IFCC) as one which will have an assay imprecision of less than $10 \%$ at the $99^{\text {th }}$ percentile of a reference population but also measure cTn in $50 \%$ or greater of a normal reference population [26]. In practice, this means that the analytical imprecision at the $99^{\text {th }}$ percentile is considerably less than $10 \%$ and often in the range of $2-5 \%$. High sensitivity
cTn (hs-cTn) assays are now available from the majority of major manufacturers [27].

The first impact of introduction of hs-cTn was a realisation that the same diagnostic efficiency could be achieved by measurement at $3 \mathrm{~h}$ from admission as was currently obtained with $6 \mathrm{~h}$ measurement. Earlier troponin detection on switching from a less sensitive to a more sensitive assay has previously been observed with iterations of assay improvement, as would be expected [28]. Early detection of troponin elevation on introduction of high sensitivity troponin assays is reflected in recommendations from formal assessment performed by health technology assessment bodies such as the National Institute for Health and Care Excellence (NICE) in the UK [29] as well guideline bodies such as The European Cardiac Society (ECS) which supported the use of measurement at admission and $3 \mathrm{~h}$ from admission [2].

The introduction of hs-cTn assays into routine clinical practice coincided with developments in the ED to explore possibilities for more rapid strategies for early categorisation of patients presenting with chest pain. This has been based around the development of accelerated diagnostic protocols (ADP) for patients with chest pain utilising scoring systems and earlier measurement of cTn [30]. This has ultimately resulted in the development of diagnostic strategies based on admission measurement of cTn using very low diagnostic cut offs either close to or at the limit of detection of the assay combined with clinical features to facilitate early rule out of AMI [31]. In addition, very short timescale repeat testing (1-2 $\mathrm{h}$ from admission) together with calculation of the rate of change of troponin (often referred to as delta troponin) has been recommended for rule-in and rule-out strategies [2, 32]. The clinical expectation therefore is that patients with suspected ACS are evaluated using high sensitivity high precision assays. Remember though that although hs assays yield equivalent clinical performance, analytical agreement is limited and absolute values from one manufacturer are not the same as those from another. This is important in examining rapid rule-out algorithms.

There have also been predictable but explicable confusions arising from the use of troponin as the gold standard and the development of hs troponin assays. The initial introduction of troponin was, arguably, oversold as a cardiac specific test for myocardial infarction. In the days of the WHO definition of AMI this was not a problem as cut offs were adopted that excluded minor troponin elevations that were, nevertheless, detectable even with the assays in use at the time [33, 34]. This situation was exacerbated when hs assays were introduced when troponin elevation due to myocardial injury in a range of clinical conditions 
was detected. The concept of myocardial injury is recognised in the current redefinition of myocardial infarction [35]. However, it must be remembered that troponin elevation, whatever the cause, predicts an adverse prognosis and that for this reason, troponin remains an excellent ED rule-out test as it identifies a low-risk group reliably.

\section{POCT in the era of high sensitivity cardiac troponin assays}

The provision of repeat testing by the central laboratory for admission and repeat measurement $1-2 \mathrm{~h}$ from admission is a significant logistic challenge and is not met by the majority of diagnostic laboratories. In addition, early studies of POCT suggest that it is the impact of having results within the timeframe of patient consultation that prompts management decisions [17]. It would therefore seem likely that provision of hs-cTn measurement by POCT is the ideal method to implement rapid diagnostic algorithms. Unfortunately, this is not yet met by any of the commercially available systems [36]. Comparable diagnostic efficiency combining a risk score with admission measurement by hs-cTn by the laboratory requires measurement $3 \mathrm{~h}$ from admission when POCT is used [37]. To date, only one analytical system suitable for near patient testing has appropriate analytical sensitivity but it is more suitable to the emergency laboratory than the true POCT environment [38]. Comparable diagnostic sensitivity to hs-cTn measurement by the laboratory is not achieved for POCT until typically $6 \mathrm{~h}$ from admission.

A range of assays are in development utilising a range of novel techniques which promise to deliver comparable analytical performance to hs-cTn measurement by the central laboratory when measured by POCT [39, 40]. There are already instruments close to routine clinical use delivering analytical performance comparable to laboratory hs-cTn measurement [41-43]. The challenge will be to validate these assays in appropriate clinical studies and ultimately by prospective randomised controlled trials.

\section{Conclusions}

Biomarker testing specifically the measurement of cTn dominates the management of patients with suspected ACS. Clinical trials performed during the early stages of POCT development when analytical sensitivity by POCT matched the central laboratory showed improvement in the TAT and shortening of ED stay. They also highlighted the importance of process in the decision-making and only demonstrated benefit when the POCT providing the analytical component was integrated within the decisionmaking pathway. The switch to high sensitivity troponin and the use of very rapid rule-out protocols have therefore been a game changer. POCT now needs to not only match the analytical capability of laboratory methods for high sensitivity troponin but also be integrated within the decision matrix to demonstrate benefit. A further caveat remains. The ED environment remains a challenging one to ensure analytical quality matches that are produced by the central lab. In addition, as with hs assays that do not give numerically equivalent results, POCT may not agree with CLT and if two different types of troponins are measured, $c \operatorname{TnI}$ in the ED and cTnT in the central lab (or vice versa), there is potential for disaster.

Author contributions: The author has accepted responsibility for the entire content of this submitted manuscript and approved submission.

Research funding: None declared.

Employment or leadership: None declared.

Honorarium: None declared.

Competing interests: The funding organization(s) played no role in the study design; in the collection, analysis, and interpretation of data; in the writing of the report; or in the decision to submit the report for publication.

\section{References}

1. Nomenclature and criteria for diagnosis of ischemic heart disease. Report of the Joint International Society and Federation of Cardiology/World Health Organization task force on standardization of clinical nomenclature. Circulation 1979;59:607-9.

2. Roffi M, Patrono C, Collet JP, Mueller C, Valgimigli M, Andreotti F, et al. 2015 ESC Guidelines for the management of acute coronary syndromes in patients presenting without persistent ST-segment elevation: Task Force for the Management of Acute Coronary Syndromes in Patients Presenting without Persistent ST-Segment Elevation of the European Society of Cardiology (ESC). Eur Heart J 2016;37:267-315.

3. Ibanez B, James S, Agewall S, Antunes MJ, Bucciarelli-Ducci C, Bueno H, et al. 2017 ESC Guidelines for the management of acute myocardial infarction in patients presenting with STsegment elevation: the Task Force for the management of acute myocardial infarction in patients presenting with ST-segment elevation of the European Society of Cardiology (ESC). Eur Heart J 2018;39:119-77.

4. Hedges JR, Gibler WB, Young GP, Hoekstra JW, Slovis C, Aghababian R, et al. Multicenter study of creatine kinase-MB use: effect on chest pain clinical decision making. Acad Emerg Med 1996;3:7-15. 
5. Stubbs P, Collinson P, Moseley D, Greenwood T, Noble M. Prospective study of the role of cardiac troponin T in patients admitted with unstable angina. Br Med J 1996;313:262-4.

6. Galvani M, Ottani F, Ferrini D, Ladenson JH, Destro A, Baccos D, et al. Prognostic influence of elevated values of cardiac troponin I in patients with unstable angina. Circulation 1997;95:2053-9.

7. Lindahl B, Venge P, Wallentin L. Relation between troponin $T$ and the risk of subsequent cardiac events in unstable coronary artery disease. The FRISC Study Group. Circulation 1996;93:1651-7.

8. Hamm CW, Heeschen C, Goldmann B, Vahanian A, Adgey J, Miguel CM, et al. Benefit of abciximab in patients with refractory unstable angina in relation to serum troponin T levels. c7E3 Fab Antiplatelet Therapy in Unstable Refractory Angina (CAPTURE) Study Investigators. N Engl J Med 1999;340:1623-9.

9. Morrow DA, Cannon CP, Rifai N, Frey MJ, Vicari R, Lakkis N, et al. Ability of minor elevations of troponins I and T to predict benefit from an early invasive strategy in patients with unstable angina and non-ST elevation myocardial infarction: results from a randomized trial. J Am Med Assoc 2001;286:2405-12.

10. Wu AH, Apple FS, Gibler WB, Jesse RL, Warshaw MM, Valdes Jr R. National Academy of Clinical Biochemistry Standards of Laboratory Practice: recommendations for the use of cardiac markers in coronary artery diseases. Clin Chem 1999;45:1104-21.

11. Myocardial infarction redefined - a consensus document of The Joint European Society of Cardiology/American College of Cardiology Committee for the redefinition of myocardial infarction. Eur Heart I 2000;21:1502-13.

12. Thygesen K, Alpert JS, White HD, Jaffe AS, Apple FS, Galvani M, et al. Universal definition of myocardial infarction. Circulation 2007;116:2634-53.

13. Luscher MS, Ravkilde J, Thygesen K. Clinical application of two novel rapid bedside tests for the detection of cardiac troponin $T$ and creatine kinase-MB mass/myoglobin in whole blood in acute myocardial infarction. Cardiology 1998;89:222-8.

14. Gerhardt W, Ljungdahl L, Collinson PO, Lovis C, Mach F, Sylven C, et al. An improved rapid troponin $\mathrm{T}$ test with a decreased detection limit: a multicentre study of the analytical and clinical performance in suspected myocardial damage. Scand J Clin Lab Invest 1997;57:549-57.

15. Heeschen C, Goldmann BU, Moeller RH, Hamm CW. Analytical performance and clinical application of a new rapid bedside assay for the detection of serum cardiac troponin I. Clin Chem 1998;44:1925-30.

16. Shah AS, Sandoval Y, Noaman A, Sexter A, Vaswani A, Smith SW, et al. Patient selection for high sensitivity cardiac troponin testing and diagnosis of myocardial infarction: prospective cohort study. Br Med J 2017;359:j4788.

17. Collinson PO, John C, Lynch S, Rao A, Canepa-Anson R, Carson $\mathrm{E}$, et al. A prospective randomized controlled trial of pointof-care testing on the coronary care unit. Ann Clin Biochem 2004;41:397-404.

18. Renaud B, Maison P, Ngako A, Cunin P, Santin A, Herve J, et al. Impact of point-of-care testing in the emergency department evaluation and treatment of patients with suspected acute coronary syndromes. Acad Emerg Med 2008;15:216-24.

19. Straface AL, Myers JH, Kirchick HJ, Blick KE. A rapid point-of-care cardiac marker testing strategy facilitates the rapid diagnosis and management of chest pain patients in the emergency department. Am J Clin Pathol 2008;129:788-95.
20. Loten C, Attia J, Hullick C, Marley J, McElduff P. Point of care troponin decreases time in the emergency department for patients with possible acute coronary syndrome: a randomised controlled trial. Emerg Med J 2010;27:194-8.

21. Goodacre SW, Bradburn M, Cross E, Collinson P, Gray A, Hall AS. The Randomised Assessment of Treatment using Panel Assay of Cardiac Markers (RATPAC) trial: a randomised controlled trial of point-of-care cardiac markers in the emergency department. Heart 2011;97:190-6.

22. Goodacre S, Bradburn M, Fitzgerald P, Cross E, Collinson P, Gray A, et al. The RATPAC (Randomised Assessment of Treatment using Panel Assay of Cardiac markers) trial: a randomised controlled trial of point-of-care cardiac markers in the emergency department. Health Technol Assess 2011;15:iii-102.

23. Tomonaga Y, Gutzwiller F, Luscher TF, Riesen WF, Hug M, Diemand A, et al. Diagnostic accuracy of point-of-care testing for acute coronary syndromes, heart failure and thromboembolic events in primary care: a cluster-randomised controlled trial. BMC Fam Pract 2011;12:12.

24. Asha SE, Cooke A, Walter E, Weaver J. Three-month outcome of patients with suspected acute coronary syndrome using pointof-care cardiac troponin-T testing compared with laboratorybased cardiac troponin-T testing: a randomised trial. Emerg Med J 2015;32:601-7.

25. Bradburn M, Goodacre SW, Fitzgerald P, Coats T, Gray A, Hassan T, et al. Interhospital variation in the RATPAC trial (Randomised Assessment of Treatment using Panel Assay of Cardiac markers). Emerg Med J 2012;29:233-8.

26. Apple FS, Collinson PO. Analytical characteristics of high-sensitivity cardiac troponin assays. Clin Chem 2012;58:54-61.

27. IFCC Committee on Clinical Applications of Cardiac Bio-Markers (C-CB). High sensitivity cardiac troponin I and T assay analytical characteristics. IFCC, 2018.

28. Melanson SE, Morrow DA, Jarolim P. Earlier detection of myocardial injury in a preliminary evaluation using a new troponin I assay with improved sensitivity. Am J Clin Pathol 2007;128:282-6.

29. Diagnostic Guidance 15 [DG15] Diagnostics Assesment Committee National Institute for Helrth and Care Excellence. Myocardial infarction (acute): early rule out using high-sensitivity troponin tests (Elecsys Troponin Thigh-sensitive, ARCHITECT STAT High Sensitive Troponin-I and AccuTnI + 3 assays). NICE, 2014.

30. Than M, Cullen L, Reid CM, Lim SH, Aldous S, Ardagh MW, et al. A 2-h diagnostic protocol to assess patients with chest pain symptoms in the Asia-Pacific region (ASPECT): a prospective observational validation study. Lancet 2011;377:1077-84.

31. Pickering JW, Than MP, Cullen L, Aldous S, Ter AE, Body R, et al. Rapid rule-out of acute myocardial infarction with a single high-sensitivity cardiac troponin T measurement below the limit of detection: a collaborative meta-analysis. Ann Intern Med 2017;166:715-24.

32. Pickering JW, Greenslade JH, Cullen L, Flaws D, Parsonage W, Aldous S, et al. Assessment of the European Society of Cardiology 0-hour/1-hour algorithm to rule-out and rule-in acute myocardial infarction. Circulation 2016;134:1532-41.

33. Collinson PO, Hadcocks L, Foo Y, Rosalki SB, Stubbs PJ, Morgan $\mathrm{SH}$, et al. Cardiac troponins in patients with renal dysfunction. Ann Clin Biochem 1998;35 (Pt 3):380-6.

34. Collinson PO, Chandler HA, Stubbs PJ, Moseley DS, Lewis D, Simmons MD. Measurement of serum troponin T, creatine kinase 
MB isoenzyme, and total creatine kinase following arduous physical training. Ann Clin Biochem 1995;32 (Pt 5):450-3.

35. Thygesen K, Alpert JS, Jaffe AS, Chaitman BR, Bax JJ, Morrow DA, et al. Fourth universal definition of myocardial infarction (2018). Eur Heart J 2018;138:e618-51.

36. IFCC Committee on Clinical Applications of Cardiac Bio-Markers (C-CB). Point of Care Cardiac Troponin I and T Assay Analytical Characteristics Designated by Manufacturer. IFCC, 2018.

37. Body R, Almashali M, Morris N, Moss P, Jarman H, Appelboam A, et al. Diagnostic accuracy of the T-MACS decision aid with a contemporary point-of-care troponin assay. Heart 2019;105:768-74.

38. Suh D, Keller DI, Hof D, von EA, Gawinecka J. Rule-out of non-ST elevation myocardial infarction by five point of care cardiac troponin assays according to the $0 \mathrm{~h} / 3 \mathrm{~h}$ algorithm of the European Society of Cardiology. Clin Chem Lab Med 2018;56:649-57.

39. Regan B, O'Kennedy R, Collins D. Point-of-care compatibility of ultra-sensitive detection techniques for the cardiac biomarker troponin I-challenges and potential value. Biosensors (Basel) 2018;8.

40. Zhou P, Liu H, Gong L, Tang B, Shi Y, Yang C, et al. A faster detection method for high-sensitivity cardiac troponinPOCT quantum dot fluorescence immunoassay. J Thorac Dis 2019;11:1506-13.

41. Mion MM, Bragato G, Casarotti A, Cosma C, Vigolo S, Vettore G, et al. Clinical performance of cardiac Troponin I: a comparison between the POCT AQT90 FLEX and the Dimension Vista analyzer in an emergency setting. Clin Biochem 2017;50:763-7.

42. Kemper DW, Semjonow V, de TF, Keizer D, van LL, Mair J, et al. Analytical evaluation of a new point of care system for measuring cardiac Troponin I. Clin Biochem 2017;50:174-80.

43. Venge $P$, van LL, Blaschke S, Christ M, Geier F, Giannitsis E, et al. Equal clinical performance of a novel point-of-care cardiac troponin I (cTnl) assay with a commonly used high-sensitivity cTnl assay. Clin Chim Acta 2017;469:119-25. 